\title{
Interfaces
}

\section{Formes, formats, espaces : du sonnet et du tanka chez Jacques Roubaud}

\section{Margaux Coquelle-Roëhm}

\section{(2) OpenEdition}

1 Journals

Édition électronique

URL : https://journals.openedition.org/interfaces/2089

DOI : 10.4000/interfaces.2089

ISSN : 2647-6754

Éditeur :

Université de Bourgogne, Université de Paris, College of the Holy Cross

\section{Édition imprimée}

Date de publication : 12 juillet 2021

ISSN : 1164-6225

\section{Référence électronique}

Margaux Coquelle-Roëhm, «Formes, formats, espaces : du sonnet et du tanka chez Jacques Roubaud », Interfaces [En ligne], 45 | 2021, mis en ligne le 12 juillet 2021, consulté le 16 septembre 2021. URL : http://journals.openedition.org/interfaces/2089; DOI : https://doi.org/10.4000/interfaces. 2089

Ce document a été généré automatiquement le 16 septembre 2021.

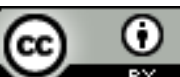

Les contenus de la revue Interfaces sont mis à disposition selon les termes de la Licence Creative Commons Attribution 4.0 International. 


\title{
Formes, formats, espaces : $\mathrm{du}$ sonnet et du tanka chez Jacques Roubaud
}

\author{
Margaux Coquelle-Roëhm
}

1 Depuis Mallarmé, le renouvellement des formes poétiques s'accompagne de nouvelles manières de penser leur inscription spatiale. Avec Un coup de dés jamais n'abolira le hasard, une nouvelle « forme du vers » conquiert l'espace visuel de la double page par «le moyen du pli central et de la césure de la page» (Murat 2005, 7-8). La mise en espace devient chez certains poètes à l'instar d'Apollinaire dans les Calligrammes, de Blaise Cendrars dans La Prose du Transsibérien ou encore de la poésie concrète, une démarche pleinement concertée. On observe ainsi une diversification des formes et des formats d'inscription du texte. Le rapport à l'espace de la page et du livre se trouve profondément transformé au $\mathrm{XX}^{\mathrm{e}}$ siècle. L'espace physique «se dégage des formats habituels et gagne en expressivité » (Chol 105). Ainsi, les « jeux d'espaces» (Chol et al.) de la poésie contemporaine invitent à s'interroger sur la disposition matérielle du poème et sur les «jeux de format» qu'elle suscite. Le format est étroitement lié aux problématiques artistiques contemporaines. David Zerbib propose ainsi de le définir comme une "construction médiatrice qui norme les modes d'inscription [...] d'usage des formes et d'informations » (Zerbib 16). Le terme s'articule aux questions d'échelle, de durée et de dimension.

2 Se présentant comme un "compositeur de mathématiques et de poésie ", le poète oulipien et mathématicien Jacques Roubaud s'est livré à une exploration des formes poétiques traditionnelles tant sur le plan théorique que par la composition - dans le cadre d'un Projet qui conjugue poésie formelle et mathématique. Le sonnet et le tanka japonais ont retenu son attention en raison de leur longévité extraordinaire. Leur inscription spatiale implique un jeu de proportionnalité avec le format de la page :

Représentons-nous un poème [...] il lui faut avant tout un calibre et un format : / le

calibre est la dose de mouvement (molécules d'air, ou lignes) prête à se voir attribuer une unité de surface, ou bouchée. / le format est une mesure légale locative de papier (Roubaud, Autobiographie, chapitre dix 157). 
3 Le poème est avant tout un mouvement de la langue, appelé à être stabilisé sur la surface d'un support déterminé par un format. La forme-poème disposée dans la page est organisée selon "des paramètres formels et même matériels » (Montémont 316). Chez Roubaud, le format est loin d'être un paramètre figé, statique: les «jeux de format » manifestent un refus de "formater » le poème, autrement dit de "mater la forme " (Sauzedde 7), cette dernière n'étant pas réductible à un unique format d'inscription. Il s'agit de rendre compte d'une pluralité de pratiques matérielles : les diverses dispositions du poème induisent des variantes de format pour agencer une même forme - voire un même poème. On observe ainsi une tension entre le choix d'un format et l'inscription variable du poème dans l'espace graphique, manifestant une " esthétique de la forme en mouvement (Puff 2009, 47). Bien plus qu'une contrainte normative, le format devient ici un dispositif matriciel à partir duquel observer la « mouvance $»^{1}$ du poème et sa circulation entre différents états, externes ou internes à la mémoire. Le mouvement de la forme engendre ainsi des variations du format d'inscription. Dans un premier temps, nous examinerons le rôle du format dans la pensée de la forme poétique chez Roubaud, avant d'articuler les «jeux de formats » avec les variations de la forme en mouvement dans ces états externes et internes à la mémoire. Enfin, nous montrerons que cette pensée de la forme conduit à concevoir le livre comme un espace multidimensionnel dont l'architecture mentale dépasse le format contraint de l'objet matériel.

\section{Les « formats des lieux géométriques des 'dépôts de langue'»}

Dès son premier recueil $€$ (1967), Roubaud pose un rapport singulier à l'espace de la page en se livrant à une « variation-distorsion » de la forme sonnet. Il agence un poème composé mentalement dans l'espace d'un support au format déterminé ${ }^{2}$. Ainsi, le format « intervient dès que s'organise ou se prépare un cadre ou un support » (Zerbib 17) comme celui de la feuille. Le sonnet est pensé comme une manière d'investir l'espace d'un support matériel. D'autre part, le poète convoque l'espace du plateau de jeu appelé go ban ( $\epsilon$, App. 4, Représentation de la partie) : chaque poème constitue un coup dans une partie de go dont l'un des modes de lecture du livre suit le déroulement. Le go ban est ainsi « vide comme une page » et un sonnet considéré comme un pion dans la partie "pourrait être matériellement, posé sur le support d'une grande feuille de papier» (Roubaud, Description du projet 35). Chaque poème était d'abord noté de manière séquentielle sur des carnets, puis disposé sur le quart de feuille $21 \times 27$, « séparé de ses congénères chronologiques [...] prêt à devenir un élément dans une structure » (Roubaud, 'le grand incendie de londres' 1700).

5 Roubaud a souligné que le choix du sonnet pour composer ce livre visait à contrer l'influence surréaliste et la «tyrannie » du vers libre : « en présentant des poèmes d'un format si désuet je ne pouvais être accusé de suivre une mode » (1648). Le glissement du terme de "forme » à celui de "format " révèle que la forme est pensée comme un

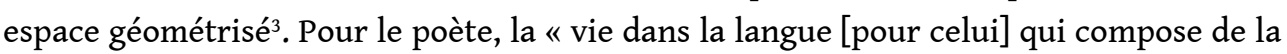
poésie comme de celui qui cherche de la mathématique » est fortement liée « au format des lieux géométriques des 'dépôts de langue' » (244) exerçant une contrainte spatiale. Il a ainsi plusieurs fois insisté sur son attachement au format «à la française » pour l'inscription manuscrite de ses poèmes. La mise en espace du sonnet est donc 
conditionnée par les mesures de la page : «Le format de la feuille, et ses diviseurs (de la moitié au seizième), était devenu le mode même de l'existence de toute langue écrite par moi, dans quelque ordre d'activité que ce soit " (244). Le poète pose en outre un rapport de proportionnalité entre les dimensions de la page et celles du poème : «le rapport de neuf à sept est, numériquement, le même que celui de la dimension longue, vingt-sept centimètres, à la dimension courte, vingt et un centimètres, des pages que j'utilisais » (244). Évoquant la généralisation du format A4 traditionnel dit "à l'américaine» (21x29,7) qu'il trouvait «infiniment disgracieux», il souligne que "l'harmonie des proportions" se trouvait «détruit par le format de la feuille 'américaine'»(244). Le sonnet est ainsi pensé comme un espace géométrique, ce «rectangle compact» aux "proportions» de «quatorze sur douze $»^{4}$ (Roubaud, Churchill 40 38), qui «a les proportions d'une page» (Roubaud, Poétique. Remarques 167, rem. 1732).

6 Plusieurs variantes-limites du sonnet sont explorées, en jouant de ce rapport de proportionnalité. Le sonnet court est emprunté au curtal sonnet de Gerard Manley Hopkins :

Dans le registre chronologique de mes sonnets, le carnet bleu, je trouve, consignée à la date du 4 décembre 1963, la trace d'une telle découverte d'objet nouveau, parent de l'objet premier, le sonnet-sphère traditionnel, découverte suivie dès le lendemain 5 décembre d'une 'invention' axiomatique [...] Hopkins écrivait : ' ${ }^{\circ} 13$ and 22 are Curtal-Sonnets, that is they are constructed on proportions resembling those of the sonnet proper, namely, $6+4$ instead of $8+6$, with however a halfline tailpiece (so that the equation is rather $12 / 2+9 / 2=21 / 2=10^{1 / 2} / 2$.' Le lendemain 5 décembre 1963 je fis un pas de plus. M'étant approprié le curtal-sonnet, je le passai en langue française sous le nom de sonnet court ('le grand incendie de londres' 1673-77) .

7 Engageant un jeu avec les "dimensions" canoniques du sonnet, le sonnet court constitue une " réduction du sonnet proper par homothétie » (Roubaud, Description du projet 34). L'examen des manuscrits ${ }^{6}$ révèle l'attention portée à la disposition de ces sonnets courts en masses respectives de 6 et 4 vers et demi séparées par une ligne de blanc $^{7}$. Contrairement aux sonnets en prose qui peuvent figurer à plusieurs sur le même feuillet, les sonnets en vers comptés sont disposés seuls dans la page - révélant ainsi qu' " un sonnet tient dans la page [...], se taille une place dans la page », est entouré de sa «mesure de blanc» (Poétique. Remarques 167). Pour le cas des sonnets courts, la réduction formelle provoque un déséquilibre visuel. Elle instaure une tension entre les dimensions du poème et le format de la page.

8 La réduction par homothétie n'est cependant pas la seule transformation observée pour passer du sonnet proper au sonnet court. Un changement affecte également la formule rimique. Les six vers venant remplacer le huitain présentent la disposition suivante: cde cde, comme le montre le premier de ces sonnets courts :

II : en contre-couleur

Je vais m'arrêter dans le noir dans le noir c

Je n'ai plus d'œil je n'ai plus de cœur chaud d

J'ai perdu le droit d'être un cœur et de battre e

Sur une porte d'aurore ah cher renard c

Et tu voudrais des rodes dans ton cachot $d$

Couche couche toi sous la ténèbre plate $\mathrm{e}$

Ne parle pas oublie pas le plus petit point a

De paix jaune tais toi l'or frotte toi d'obscur $b$

Arrache de ton champ le chiendent d'azur $b$ 
Va et la nuit bientôt te pèsera moins a

Que tes jours reçurent $(\in 81) \mathrm{b}$

Le passage au sonnet court supposerait un retournement des schémas rimiques respectifs des quatrains et des tercets. Ainsi, «au mouvement de l'homothétie s'est ajouté en fait un renversement, un retournement qui échange [...] les positions respectives des 'quatrains' et 'tercets' de départ ». Il s'agit d'une double « distorsion » non seulement de réduction proportionnelle - mais aussi «d'ordre conceptuel $»^{8}$, faisant éprouver au poète "l'élasticité axiomatique" de la forme ("le grand incendie de londres' 1676).

Jacques Roubaud s'est également tourné vers la tradition japonaise médiévale du tanka - découvert à l'occasion de lectures sur le jeu de go - dès son deuxième recueil (Mono no aware), montage de poèmes empruntés aux grandes anthologies impériales. La forme compte 31 syllabes métriques décomposées en segments de 5-7-5-7-7 syllabes. Le poète reconnaît une sorte de parenté entre le sonnet et le tanka en raison de leur compacité et de leur impartibilité. Il établit aussi un parallèle concernant leur devenir dans la tradition ('le grand incendie de londres' 1882-83). Pour explorer le format d'inscription du tanka, intéressons-nous plus particulièrement au troisième livre de poésie publié par Roubaud, Trente et un au cube, qui fut composé entre 1971 et 1972 et publié en 1973 chez Gallimard. Le livre marque le troisième moment d'une exploration du changement des formes envisagée dans une perspective diachronique (Puff 2009, 333 sq; 345 sq). Si le tanka repose sur une formule métrique de 31 syllabes, l'originalité de la tentative repose sur le fait de la projeter dans trois dimensions. Chaque poème compte 31 "vers" dont chacun compte 31 syllabes métriques, décomposée selon l'addition suivante : 5+7+5+7+7. Ce découpage intervient à tous les niveaux du recueil : au niveau de la ligne, dont chaque segment de cinq ou sept syllabes est marqué par un espacement interne; au niveau de la cobla (strophe) composée de cinq ou sept lignes délimitées par des blancs interlinéaires ; au niveau du poème et enfin, au niveau du recueil, qui comporte 31 poèmes :

Il y a, ainsi, cinq subdivisions du livre : la première de cinq poèmes, la deuxième de sept poèmes...; il y a cinq «strophes " en chaque poème : la première de cinq « vers », la deuxième de sept ... ; cinq « segments » (hémistiches) en chaque « ligne » (vers): la première de cinq "positions» (voyelles comptées) [...]. En outre, une division supplémentaire oppose une première partie (un «hokku») de 5-7-5 à une seconde (7-7). (Description du projet 78)

Le livre est ainsi présenté comme "un poème qui est trente et un poèmes ». La référence au nombre permet de penser le format dans son caractère bidimensionnel, celui de la page, mais aussi tridimensionnel, celui du recueil conçu comme «cube »: « l'unité du livre se cherche sur trois dimensions (cube de 31)» (78).

Cette pensée de la forme comporte des implications matérielles et symboliques. Au niveau matériel, le déploiement de la formule métrique conduit à créer un objet-livre singulier, imposé par l'inscription spatiale de la forme. Chaque tanka se déploie sur une feuille disposée en format paysage. Celle-ci doit être dépliée dans la largeur pour dévoiler le poème et invite le lecteur à un geste de manipulation. Le poète raconte en effet que ce format singulier s'est imposé a posteriori :

Le livre que j'ai écrit en 1972, Trente et un au cube, est un «format » impossible, un format de compensation, qui force le poème à s'allonger horizontalement sur deux pages. Voilà une justification de sa «métrique » qui m'avait échappé, à l'époque ('le grand incendie de londres' 694-95). 
13 Le choix métrique aurait ainsi conduit à une réflexion sur le format d'inscription et la matérialité physique $\mathrm{du}$ volume. L'adoption $\mathrm{du}$ format oblong découlerait $\mathrm{du}$ déploiement de la formule métrique. Le format " paysage » n'en est pas moins apparu tôt dans la genèse de l'œuvre comme un moyen de "faire tenir » dans la page les 31 syllabes métriques. En 1972, Roubaud publie en avant-première un fragment de Trente et un au cube sur une feuille dépliante (Roubaud "Poèmes", cité par Di Manno et Garron 150). Il rappelle avec humour dans son entretien avec Jean-François Puff les «quelques grognements» qui ont marqué l'accueil du livre de la part du service de fabrication, " puisqu'il a fallu faire des pages dépliantes» (Roubaud et Puff 105). Le format paysage offre un gain dimensionnel considérable et garantit l'identité visuelle du poème dans la page.

14 Au niveau symbolique, le format dialogue avec le thème du livre. Ainsi, le poème d'amour entrelace de manière fréquente un commentaire sur son inscription spatiale (Fig. 1) :

Figure 1. Jacques Roubaud. Trente et un au cube. Paris : Gallimard, 1973, p. 21.

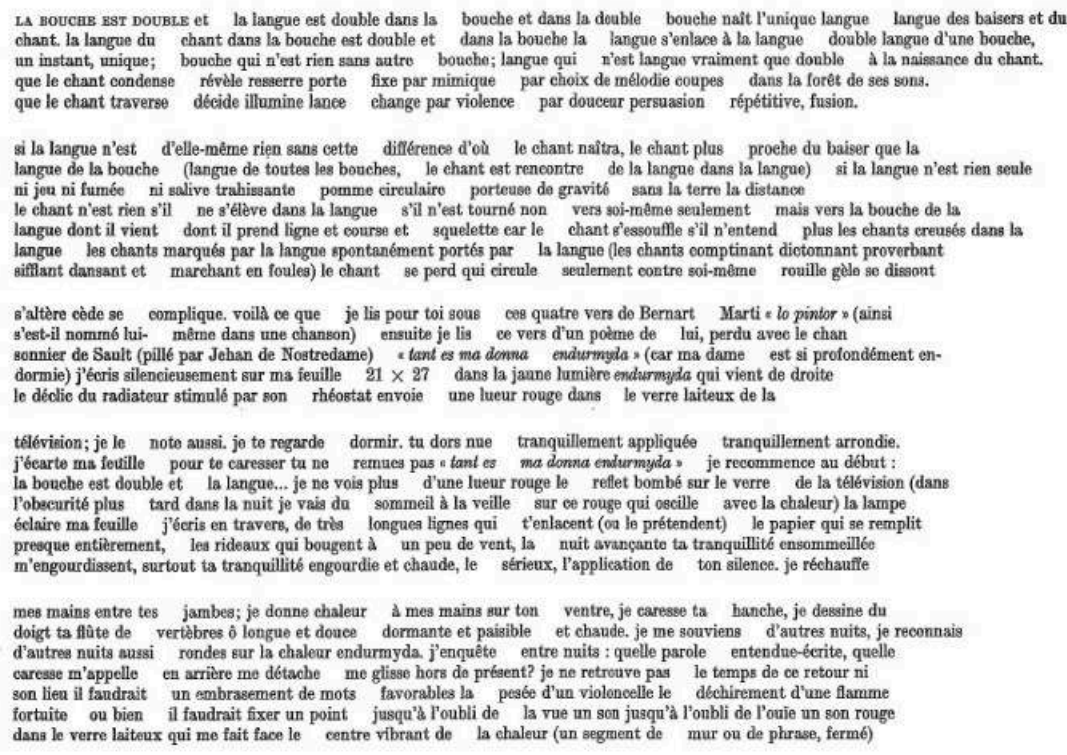

15 L'espace concret dans lequel le poème s'inscrit se trouve réintégré au sein des isotopies textuelles. La parole d'amour s'entrelace autour du corps de la femme aimée dans la stanza - chambre et strophe (Agamben 4) - où résonne le poème. Le modèle du cube, comme nous aurons l'occasion de le montrer, "fait du livre un espace où le poète enferme par la parole celle qu'il aime » (Montémont 351).

Un rapport de proportionnalité avec le format de la page s'observe également si l'on tient compte, comme pour le sonnet, des lignes de blanc séparant les différentes strophes : les trois premières étant séparées de deux lignes de blanc $(5+7+5+2=19)$ et les deux dernières par une ligne $(7+7+1=15)$, on parvient alors à un rapport de 19/15, quasiment égal à celui de 27/21 - lui-même triple de 9/7 (les dimensions du sonnet) (Puff 2009, 362). Le manuscrit offert comme don amoureux à Florence Delay est inscrit sur de grandes feuilles de papier canson d'un format différent. Leur disposition convoque cependant le même rapport de proportion (en tenant compte des blancs 
entre les coblas $)^{9}$. Le jeu de proportion marque le parallèle que Roubaud établit entre les deux formes et le format paginal. Il réalise cette synthèse de formes qui caractérise le déploiement du Projet de poésie. Il est cependant important de remarquer que le rapport de proportionnalité implique une torsion dans la pensée de la forme. En effet, il ne s'établit pas entre les longueurs et "largeurs" (nombre de syllabes du vers) respectives du poème et de la page, mais entre les "dimensions" respectives des constituants strophiques hiérarchisés $-(4+4)+(3+3)$, pour le sonnet $;(5+7+5)+(7+7)$, pour le tanka -, en comptant les lignes de blanc, par rapport au format de la page. On peut d'emblée remarquer l'écart possible entre un modèle mental et le format de l'espace matériel. Pour le tanka, le modèle d'un carré de 31 (vers) x 31 (syllabes) - lié aux dimensions de la forme - entre en tension avec celles de la page.

La page 21x27 étant conçue comme un espace matriciel, l'adoption du format découlerait d'une exploration de la forme appuyée sur sa formule métricoarithmétique. Il constitue donc un «opérateur clé des pratiques matérielles et symboliques " (Zerbib 16) liées à la spatialisation du poème. L'espace du support interagit avec les propriétés structurelles de la forme et sa potentialité pour les mettre en scène.

Toutefois, la circulation du poème d'un support à un autre induit des tensions dans les rapports de proportionnalité précédemment évoqués pour l'écriture manuscrite. Par exemple, les sonnets de $\epsilon$ peuvent être disposés à plusieurs sur une même page imprimée, là où le manuscrit privilégiait l'identité visuelle forte du sonnet dans la page. Le livre imprimé tend donc à normer ou restreindre les jeux de formats et de proportionnalité évoqués jusqu'ici. Le poète a ainsi déploré la distance creusée entre sa "'conception' [de la forme] appuyée sur la page manuscrite" ("le grand incendie de londres' 244) et le livre imprimé. Or, la forme est pour Roubaud une affaire de mouvement et de variation visant à contrer le figement du poème dans sa seule forme écrite : les « différentes façons de poser [le poème] sur la page sont des variantes d'un même objet de pensée » ("La chose la plus importante à dire d'un poème ». Repris dans 'le grand incendie de londres' 1738). Il s'agit dès lors d'inscrire la question du format dans un réseau de relations réciproques entre différents états du poème - impliquant une temporalité propre.

\section{Jeux d'espace, « jeux de format » et « forme en mouvement »}

\section{Un « quatuor de formes »}

Dans un entretien, Roubaud soulignait que «simplement par ce qui est dit et la façon dont c'est posé sur le papier, on peut faire intervenir un nombre considérable de dimensions, finalement presque plus que dans un tableau» (Bouhénic). Son apport théorique original est de penser le poème comme un "quatuor de formes ". Sur le modèle de la canso des troubadours, qui entrelace mots et sons, écriture et musique (Roubaud, «Poésie et oralité » 307), la poésie serait ainsi destinée à un " œil-oreille » (Roubaud, Poésie, etcetera, ménage 126) - supposant que l'on se place du point de vue de la réception du poème par le lecteur ou auditeur. Cette "hypothèse de la poésie » est présentée pour la première fois dans Poésie, etcetera ménage, puis affinée au fil de ses travaux théoriques notamment dans Poétique. Remarques (Rem. 3345-3348; 4002-4005). 
Pour lui, tout poème existe en une «quadruple forme » (361, rem. 4002), composée de deux couples formels, l'un externe et l'autre interne. Les deux états externes sont la forme orale et écrite du poème et constituent une "partition». Ces deux formes externes peuvent parfois entretenir des «rapports conflictuels» qui fondent la « composante rythmique de la poésie » (307). Le poète insiste sur leur variabilité :

4004. Hypothèse des poèmes: les formes d'un poème bougent. Il n'y a donc pas de forme fixe et définitive d'un poème quel qu'il soit.

4005. La variabilité des formes externes d'un poème est grande : disposition sur la page, performance orale, bougé de l'orthographe, de la typographie (361).

Les « jeux de format » participent donc de cette variabilité. Roubaud considère ainsi qu' " un poème change si on change le format de sa mise en page » (260). Le choix d'un format permettrait de stabiliser une version du poème sur la page, un état provisoire du poème en attente d'être remis en jeu. Ainsi, «le format importe en ce qu'il fait de l'espace de la feuille un lieu où blancs et noirs de lettres imprimées composent, composent ensemble et composent quelque chose qui 'prend' sur la feuille, qui s'y stabilise » (Moncond'huy 2011, 282). Déterminer le format de la page permet de définir les conditions d'expérience d'une forme-espace en jouant de ses dimensions.

D'autre part, tout poème comporte une existence interne à la mémoire de celui qui la reçoit : celle-ci est à la fois « éQrite » - soit « l'image visuelle intérieure, mémorielle, de la page de poésie » - et " aurale », par son existence sonore dans la mémoire intérieure. L'existence d'une page mentale de poésie permet une visualisation intérieure de sa spatialité, selon une configuration visuelle " photographiée " par la mémoire. La forme aurale du poème conduit au déploiement de la mémoire du poème dans un "espace sonore intime" (Murat). Roubaud insiste sur l'importance des formes internes, qui contrairement aux formes externes, sont toujours réalisées. Ainsi, le poème ne peut être réduit à sa forme externe écrite qui est « oisive », c'est-à-dire figée, immobilisée dans la page :

Ce n'est qu'en entrant dans la mémoire intérieure de qui le reçoit et fait sien qu'il accède à un nombre de dimensions respectable, qu'il devient vraiment poème, et plus une partition [...]. Sans la mémoire intérieure, sans mémoire de poésie, la page est immobile et la voix éphémère (Poésie, etcetera, ménage 128).

La réception intérieure du poème garantit le déploiement de l'ensemble de ses dimensions, ainsi que son mouvement dans la mémoire intérieure. Alors que les formes externes sont interpersonnelles, celles internes sont essentiellement idiosyncrasiques, « incommunicables d'une tête à l'autre; elles sont toujours en mouvement dans les mémoires, dans les pensées » (Poétique. Remarques 307). Roubaud met ainsi l'accent sur le processus de mémoire que le poème met en mouvement quand il «entre dans la tête ", et sur l'usage intime que celui-ci peut comporter (Puff 2018).

Concernant le sonnet court, le poète évoque la page mentale (éQrite) dans laquelle se joue le rapport de réduction par homothétie: «on crée de cette manière un nouvel espace, un nouveau plan mental vertical, où on place une nouvelle feuille, tout aussi mentale, pour y déposer le poème nouveau " ('le grand incendie de londres' 1675). Ainsi, «il y a à la fois au moins homothétie, renversement et mise en évidence d'une troisième dimension des poèmes, hors la page, dans le champ mental où travaille la mémoire de poésie » (1677). La prise en compte de la forme éQrite permet ainsi de faire intervenir une dimension supplémentaire du poème, pour garantir la variation et le mouvement de la forme-sonnet. Les tensions entre les dimensions de la forme et le format du support sont donc relatives à cette pensée de la forme en mouvement : tel le 
« couteau de Lichtenberg », le modèle conceptuel de la forme se maintient au sein de diverses variations, grâce au mouvement des formes internes à la mémoire. Tout poème porterait ainsi, en "arrière-page » (1890), une forme abstraite virtuelle qui en est la matrice potentielle.

\section{« Pseudo-tanka », « tanka fantôme »}

Pour mieux circonscrire ce rapport dynamique entre les formes internes du poème et le format de l'espace matériel, nous pouvons nous intéresser au projet multidimensionnel ayant impliqué une artiste peintre et cinq écrivains. Pour Mille e tre, Micaëla Henich a fait mille et trois dessins à l'encre de Chine. Elle en a confié deux cents à chacun des cinq auteurs suivants : Jacques Derrida, Tom Raworth, Dominique Fourcade, Michael Palmer, et Jacques Roubaud afin qu'ils les légendent. Tous les livres issus de ces collaborations sont de formats différents tandis que les dessins formant un rectangle vertical ont toujours la même dimension $(6 \times 16 \mathrm{~cm})$. Michael Palmer a choisi un format oblong $(9,3 \times 28 \mathrm{~cm})$ pour proposer des vers sériels, sur le modèle $\mathrm{du}$ " moulin à prière " qui déroule les mantras des prières bouddhistes.

Dans 200 flèches (Roubaud et Henich), Jacques Roubaud a choisi la matrice formelle du tanka pour accompagner les dessins 201 à 400 qui occupent approximativement les $2 / 3$ de la page. Un tanka légende ainsi chaque dessin pour faire se rencontrer langage verbal et langage plastique. Le format hors-norme adopté $(24 \times 14 \mathrm{~cm})$ participe de l'hybridation des formes plastiques dans un espace tissé d'interactions réciproques. L'écriture (en relation avec le langage idéogrammatique) relaye le mouvement du dessin. Le poète s'est inspiré du tir à l'arc, et plus particulièrement d'un ensemble de poèmes didactiques japonais $\mathrm{du} \mathrm{XVI} \mathrm{I}^{\mathrm{e}}$ siècle énonçant les " principes à la fois techniques (abstraits), et moraux » ('le grand incendie de londres' 658) de cet art. Le « je » qui apparaît à partir du poème 204 est la flèche elle-même. L'isotopie du mouvement présente dans de nombreux poèmes renvoie autant à celui de la flèche qu'aux modalités d'inscription spatiale du tanka (Fig.2) : 
Figure 2. Mille et tre, deux. 200 flèches, dessin 209 et sa légende. Encre sur papier, 6 x 16.

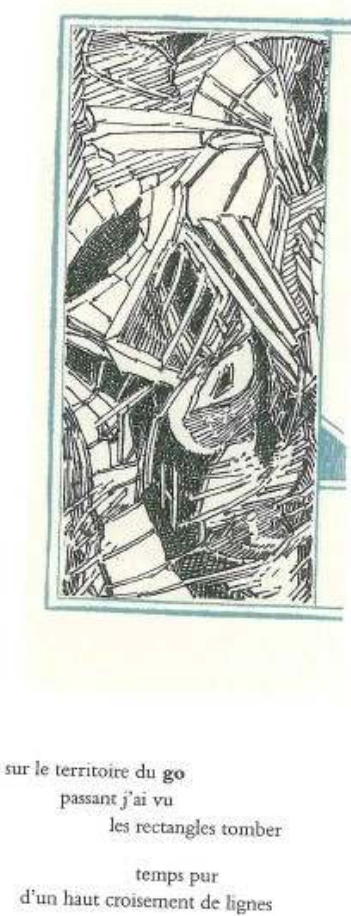

(c) Michaëla Henich et Théâtre Typographique, 1995. Tous droits réservés. lecteur -, la disposition dans la page et le format de l'espace matériel. À deux reprises, Roubaud emploie le terme de "pseudo-tankas» pour évoquer ces poèmes. La disposition qu'il choisit fait en effet entorse au découpage métrique classique (5-7-5-7-7) puisqu'il répartit les cinq vers en deux strophes séparées d'une ligne de blanc, de trois et deux vers respectivement, et « permute la disposition des vers dans la page » (Consenstein 169). On peut ainsi observer différentes variations d'investissement de l'espace graphique, les cinq vers pouvant commencer « en des points distincts de la ligne » ('le grand incendie de londres' 1889), selon un système de permutation concerté. Roubaud explique qu'à chacun des pseudo-tankas "est associé en arrière-page, filigrane, ou trame d'un dessin, un véritable tanka fantôme de langue française » (1890). Il agence le poème en comparaison avec un " poème virtuel ", « en arrière-plan du poème présenté ", où les vers ont la «mise en place standard » (1890), selon le modèle suivant (Fig.3 et 4) : 
Figure 3. Disposition standard des vers.

Vers 1

Vers 2

Vers 3

Vers 4

Vers 5

Figure 4. Mille et tre, deux. 200 flèches, dessin 248 et sa légende. Encre sur papier, 6 x 16 .

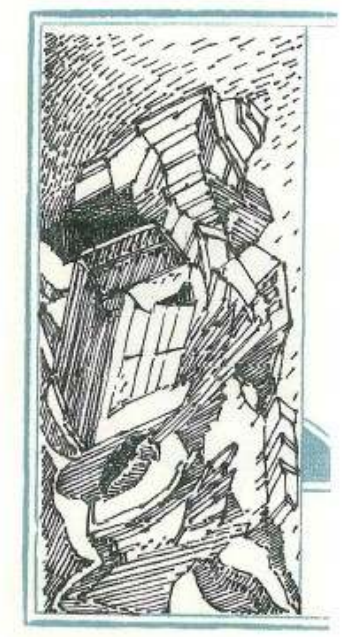

248. il y a ces masses de rectangles

qui pèsent dans un resserrant espace

il n'y a d'imaginable

que ces masses d'air quadrille

et ce sol serrant l'espace

(c) Michaëla Henich et Théâtre Typographique, 1995. Tous droits réservés.

Le motif de la trame et du dessin est emprunté aux grandes anthologies japonaises. Transposé ici, il suggère l'idée qu'une page éQrite reprenant la disposition «standard ${{ }^{10}}^{10}$ u tanka japonais s'inscrit en palimpseste du «tanka-fantôme de langue française " disposé dans la page. De la même manière, Roubaud soulignait pour le sonnet court que «le 'sonnet propre' est présent, en arrière » ('le grand incendie de londres' 1677). Il y aurait donc une asymétrie possible entre la dimension matérielle et mentale des formes visuelles, permettant de prêter la forme à diverses variantes spatiales. De même, certains poèmes ne respectent pas le décompte des 31 syllabes métriques ni leur découpage en 5 vers de 5-7-5-7-7, comme dans l'exemple suivant (Fig. 5) : 
Figure 5. Mille et tre, deux. 200 flèches, dessin 284 et sa légende. Encre sur papier, 6 x 16.
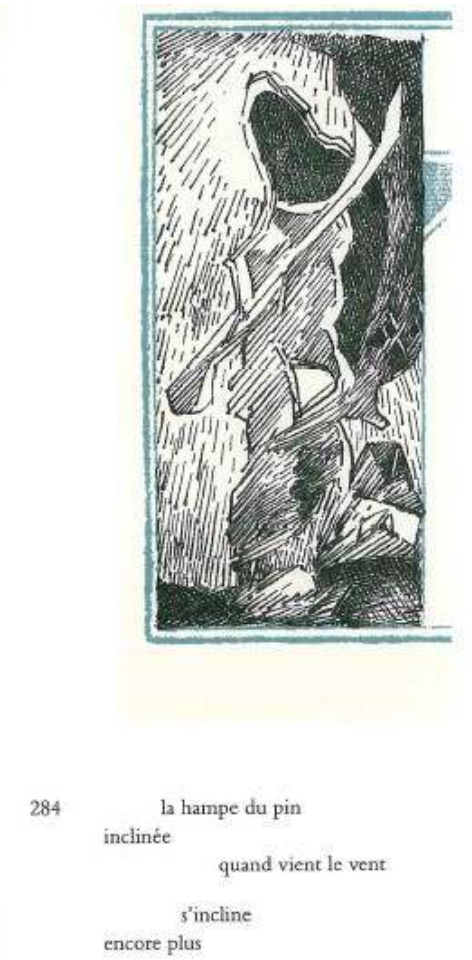

(c) Michaëla Henich et Théâtre Typographique, 1995. Tous droits réservés.

29 Ces variantes métriques illustrent la disjonction possible entre un format métrique «standard » et les variations créatives auxquelles la forme est soumise au gré de ses actualisations. Elles manifestent ainsi une mémoire flottante des formes, telles que celles-ci sont inscrites dans la mémoire intime d'un sujet selon une sorte de "format fantôme ». La spatialité du poème s'articule ainsi à la temporalité singulière que suppose une profération aurale : « une durée, métronomique selon la mesure, doit être 'entendue' en même temps que les syllabes effectives (vues-ouïes), qu'une performance orale pourrait manifester par ralentissements et accélérations " ("le grand incendie de londres' 1890). Il peut donc exister un écart entre les formes aurales et éQrites du poème, singulières et propres à chaque lecteur. Dès lors, la notion de format ne renvoie plus seulement aux dimensions du support et à ses propriétés matérielles, mais aussi à la durée rythmique du déploiement sonore du poème dans la mémoire.

\section{Échapper au format, entrer dans une quatrième dimension?}

Une telle conception de la forme en mouvement pourrait laisser penser que celle-ci en viendrait à " échapper au format ", de par sa double existence - externe et interne. Penser le format d'inscription (pour l'œil) et la durée interne à la profération (pour l'oreille) invite à les examiner à l'aune de modèles spatiaux qui régissent la construction du livre.

On a pu observer une revalorisation du caractère pluridimensionnel de l'ouvrage : comme nous l'avons montré, la pensée du format s'articule à une pensée de l'espace du 
livre $^{11}$. Ce qui importe à Roubaud, c'est « d'ajouter un étage supplémentaire à celui du poème [...] celui du livre » (Description du projet 69), idée qu'il généralise à partir des principes de montage des poèmes mis en œuvre dans les anthologies impériales médiévales japonaises ( Sur le Shinkokinshū»). Dans $\epsilon$, la réflexion sur l'inscription du poème dans la page est étroitement liée à une réflexion sur « la fabrication du livre qui devait les contenir et les agencer" selon des opérations de "construction et de groupement" ('le grand incendie de londres' 244). Le livre comporte une "cohérence imaginaire, macroscopique ", projeté comme "sonnet lui-même " (Description du projet 36). Chaque partie ou paragraphe du livre abordé dans sa progression linéaire forme ainsi un "sonnet de paragraphes" faisant du livre un "sonnet de sonnets" ("le grand incendie de londres' 1695). La structure de Trente et un au cube est également pensée comme celle d'un "poème de poèmes » (Description du projet 69). Le titre montre bien que le livre est un volume, la référence au nombre permettant d'appréhender l'espace du recueil comme un espace en trois dimensions. Les métaphores spatiales s'avèrent pertinentes pour penser l'architecture même du livre : «la page ajoutée, si on la lève avec l'adjacente à la verticale, forme avec la première trois côtés d'un quasi-cube " (Cardonne-Arlyck 385 ; Montémont 351), désigné dans le poème comme "cube de vent », « cube de sel », « cubes de fil » (Trente et un au cube 53). Le livre épouse en outre une "'macroscopie' circulaire [...] le trente et unième 'poème', dans sa dernière 'moitié', reprenant le début » (Description du projet 78). Le poète reprend en cela une intuition des grandes anthologies impériales liant les poèmes en chaînes, selon des méthodes d'intégration et de progression (Konichi). La structure interne du livre n'est plus seulement cubique, mais circulaire. Elle évoque l'écoulement d'une année et le temps cyclique des saisons par la reprise d'image-clefs. Le poème "vise à une reconfiguration du temps, dont la circularité est l'un des modes » (Puff 2009, 353). Nous pouvons donc distinguer le livre, conçu par le poète comme une forme mentale sur le modèle d'un " poème de poème » doté d'une organisation interne réfléchie, de l'objet matériel au format contraint. Cette distinction entre un modèle spatial interne et un format externe permet d'éclairer à nouveaux frais les modèles spatiaux qui régissent la conception du volume.

Roubaud travaille ainsi à déplacer, réinventer les métaphores spatiales de la forme : le sonnet est comparé à une «sphère mathématique » ('le grand incendie de londres' 1672). Cette métaphore peut sembler surprenante si l'on s'en tient à sa forme externe et matérielle. Le sonnet est tout sauf une forme circulaire, et l'on pourrait jouer du motif de la flèche pour décrire la tension vers sa pointe finale. Une telle conception aboutit certes à une forme de circularité mais au prix peut-être, d'un enjeu spécifique à la forme même du sonnet. Ici, il ne serait plus pensé dans sa verticalité, mais comme un " quasi-cristal $»^{12}$ - espace à plusieurs dimensions conçu à l'aune de sa forme interne. Dès lors, cet espace excéderait la seule question du format pour tendre vers une pensée de la forme-volume.

Ces modèles spatiaux circulaires rappellent la «Grande Feuille Mentale » conçue dans « une pièce circulaire " ('le grand incendie de londres' 287-88; 619). Roubaud se place au milieu de cet espace figurant celui du livre pour contempler la prose de mémoire se déployer en une longue ligne unique. Il a sans cesse retravaillé ce modèle spatial en volume, jusqu'à proposer l'idée d'un « cube » de mémoire :

856 J'ai fait parfois aussi l'expérience d'une mise en boîte de la mémoire. D'une chambre, toujours, on est en droit de faire un cube, un cube mémoriel. Ou un parallélépipède. Un sonnet, par exemple, convient aux quatre murs verticaux. On 
compose le premier quatrain, qu'on met ensuite derrière sa tête. [...] On place le deuxième quatrain sur le mur qui fait face, le premier tercet sur le mur de droite, le deuxième sur le mur de gauche, entre les deux fenêtres (La Dissolution 409). utilisée comme lieu de composition et de disposition du poème, formant également une chambre d'écho permettant la rumination « aurale » du poème. La forme est davantage pensée comme une "clôture", un lieu érémitique dans lequel méditer. "Espace et volume ", elle prend "toutes ses 'dimensions' » dans un espace mental qui la rend disponible au travail du temps (Moncond'huy 2011, 283). Le sens de ces métaphores spatiales ou architecturales est à trouver du côté des arts de mémoire, bien connus de l'auteur. Roubaud s'inspire du modèle du «théâtre de mémoire» de Camillo pour formuler l'hypothèse d'un "champ mnémonique ", géométrisé et multidimensionnel où stabiliser les images mémorielles (L'Invention $d u$ fils de Leoprepes 72-74). Il déplace ce principe optique pour se placer au centre du dispositif. Il convoque un espace mental dans lequel s'enfermer, faisant du poème un espace à parcourir, pour accomplir un protocole de méditation passant par la visualisation, la profération, la répétition et la variation.

Cet espace à plusieurs dimensions est décrit comme un «lieu dans un compact dans une boîte à $\mathrm{N}$ dimensions " (Trente et un au cube 69), dans le seizième poème du livre (Fig.5) :

Figure 6. Jacques Roubaud, Trente et un au cube. Paris : Gallimard, 1973, p. 69.

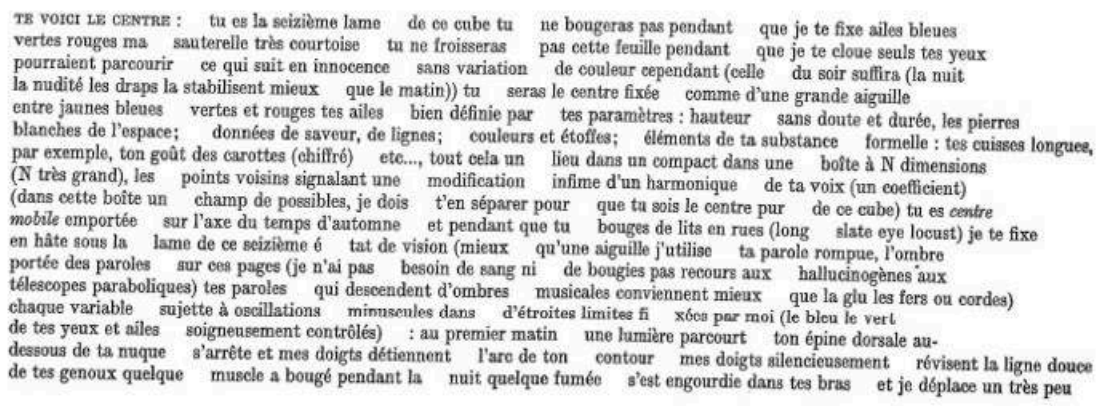

La disposition en deux blocs de 17 et 14 lignes séparés de blancs manifeste visuellement le centre exact du livre. Le poème mentionne les «différents axes permettant de construire un cube " (Montémont 352) et lui projette une quatrième dimension, celui de la «durée » appelée aussi « axe du temps ». Le poème constitue alors cet « espacetemps-forme" évoqué plus loin dans le poème pour former un hyperespace multidimensionnel. L'enjeu serait alors de «discipliner le temps », par le rythme et la 
durée de déploiement du poème dans la voix. Cet enjeu apparaît comme le résultat d'une méditation passant par la forme $(\in 18)$, impliquant la contemplation et la répétition. Le mouvement de la forme permettrait un retour à la mezura, accomplissant un enjeu tout à la fois esthétique et éthique.

En conclusion, l'étude de l'articulation entre "jeux d'espaces » et jeux de formats dans l'exploration des formes chez Jacques Roubaud a permis de mettre en relief deux enjeux complémentaires. D'une part, l'exploration formelle, liée à une conception oulipienne de la potentialité d'une forme, agit sur les dimensions du poème (un " format métrique ») voire sur le format du support, jusqu'à introduire un rapport de proportion ou une tension visuelle et spatiale. D'autre part, la circulation d'un même poème entre différents états constitutifs du "quatuor de formes» (externes ou internes) en fait une "variante perpétuelle de [lui-même]» (Roubaud, Dors 19). Dès lors, le format, comme lieu de « dépôt » de la forme écrite externe, constitue un des paramètres de variabilité de la forme. Les variétés de supports et de formats investis visent ainsi à "n'immobilis[er] aucun poème " (19). Si la forme persiste dans ses différents formats d'inscription, la variation du format induit une spatialité du poème, un rythme graphique et une temporalité de lecture différentes. Du reste, la prégnance des modèles spatiaux pluridimensionnels et en volume a montré que la question du format ne se restreint pas aux dimensions d'un support. Elle fait intervenir la notion de durée propre au déploiement du poème dans la mémoire de celui qui le reçoit dans sa forme aurale. Voué à résonner dans un espace mental méditatif selon une durée façonnée par le rythme, le poème devient alors - au gré de ses variations successives une forme vivante de mémoire.

\section{Ouvrages cités}

AGAMBEN, Giorgio. Stanze : parole et fantasme dans la culture occidentale. Trad. Yves Hersant. Paris : Payot \& Rivages, 2009.

BOUHENIC, Pascale. L'atelier d'écriture de Jacques Roubaud. Paris : Centre Georges Pompidou, 1995.

CARDONNE-ARLYCK, Elisabeth. Véracités : Ponge, Jaccottet, Roubaud, Deguy. Paris : Belin, 2009.

CHOL, Isabelle. « Configurations poétiques et espace matériel du livre d'avant-garde ». Formes: supports / espaces. Dir. Christelle Reggiani, Christophe Reig, Hermès Salceda et Jean-Jacques Thomas. New York : Presses Universitaires du Nouveau Monde, 2015. 105-120.

CHOL Isabelle, Bénédicte MATHIOS et Serge LINARES (dir.). LiVres de pOésie, Jeux d'eSpace. Paris : H. Champion, 2016.

CONSENSTEIN, Peter. « Tanka encre ». Formes : supports / espaces. Dir. Christelle Reggiani, Christophe Reig, Hermès Salceda et Jean-Jacques Thomas. New York : Presses Universitaires du Nouveau Monde, 2015. 157-177. 
DI MANNO, Yves et Isabelle GARRON. Un nouveau monde. Poésies en France, 1960-2010 : un passage anthologique. Paris : Flammarion, 2017.

HOCQUARD, Emmanuel. «Les dernières nouvelles de ma cabane, nº, 28 novembre 1997 ». Repris dans Ma haie. Paris : P.O.L, 2001. 427-428.

HOPKINS, Gerard Manley. Poems of Gerard Manley Hopkins. 3e edition. Oxford : Oxford University Press, 1949.

KOBLE, Nathalie et Mireille SEGUY (dir.). Jacques Roubaud médiéviste. Paris : H. Champion, 2018.

KONICHI, Jinichi. « Association and progression : principles of integration in anthologies and sequences of Japanese court poetry ». Harvard Journal of Asiatic Studies 21 (1958) : 67-127. Trad. Robert H. Browner et Earl Miner.

MONCOND'HUY, Dominique. « D'Aragon à Roubaud et Hocquard : le sonnet comme espace ». Le sonnet contemporain : retours au sonnet (Actes du colloque « Retours au sonnet » organisé à l'Université de Poitiers les $1^{\text {er }}$ et 2 septembre 2007). Dir. Dominique Moncond'huy et Alain Chevrier. Formules / Revue des créations formelles 12 (2008) : 11-21.

MONCOND'HUY, Dominique. «Sonnet et entreprise de mémoire ». Jacques Roubaud : compositeur de mathématique et de poésie. Dir. Agnès Disson et Véronique Montémont. Nancy : Absalon, 2011. 279-292.

MONTEMONT, Véronique. Jacques Roubaud : l'amour du nombre. Villeneuve d'Ascq : Presses Universitaires du Septentrion, 2004.

MURAT, Michel. « Entre l'œil et l'oreille ». Théories du lyrique. Une anthologie de la critique mondiale de la poésie, 2020. URL : http://lyricalvalley.org/blog/2020/02/13/entre-loeil-et-loreille/ (page consultée le 13 mai 2021).

MURAT, Michel. Le Coup de dés de Mallarmé: un recommencement de la poésie. Paris : Belin, 2005.

NOEL, Bernard. L'Espace du poème. Entretiens avec Dominique Sampiero. Paris : P.O.L, 1998.

PITCHFORD, Lois W. « The Curtal Sonnets of Gerard Manley Hopkins ». Modern Language Notes 67:3 (1952) : 165-169.

PUFF, Jean-François. Mémoire de la mémoire : Jacques Roubaud et la lyrique médiévale. Paris : Classiques Garnier, 2009.

PUFF, Jean-François. « Jacques Roubaud et l'usage méditatif du poème ». Poésie moderne et méditations. Actes des journées d'étude organisées à l'Université de Rouen les 21 mars 2017 et 19 mars 2018. Dir. Christophe Lamiot et Thierry Roger. Publications numériques du CEREdI 21 (2018). URL : http://ceredi.labos.univ-rouen.fr/public/?jacques-roubaud-et-l-usage.html (page consultée le 13 mai 2021).

ROUBAUD, Jacques. $\in$. Paris : Gallimard, 1967.

ROUBAUD, Jacques. « Sur le Shinkokinshū, huitième anthologie impériale japonaise ». Change 1 (4e trimestre 1968) : 73-106.

ROUBAUD, Jacques. Mono no aware : le sentiment des choses. Cent quarante-trois poèmes empruntés au japonais. Paris : Gallimard, 1970.

ROUBAUD, Jacques. « Poèmes ». Les Cahiers du Chemin 15 (mai 1972) : n.p.

ROUBAUD, Jacques. Trente et un au cube. Paris : Gallimard, 1973. 
ROUBAUD, Jacques. Autobiographie, chapitre dix : poèmes avec des moments de repos en prose. Paris : Gallimard, 1977.

ROUBAUD, Jacques. Dors ; (précédé de) Dire la poésie. Paris : Gallimard, 1981.

ROUBAUD, Jacques. L'invention du fils de Leoprepes : poésie et mémoire. Saulxures : Circé, 1993.

ROUBAUD, Jacques. Poésie, etcetera, ménage. Paris : Stock, 1995.

ROUBAUD, Jacques. « La chose la plus importante à dire d'un poème, c'est : 'apprenez-le', entretien avec Macha Séry ». Le Monde de l'éducation 288 (janvier 2001) : 14-19.

ROUBAUD, Jacques. Churchill 40 et autres sonnets de voyage : 2000-2003. Paris : Gallimard, 2004.

ROUBAUD, Jacques. La Dissolution. Caen : Nous, 2008.

ROUBAUD, Jacques. 'le grand incendie de londres'. Paris : Seuil, 2009.

ROUBAUD, Jacques. Quasi-cristaux : un choix de sonnets en langue française de Lazare Carnot (1820) à

Emmanuel Hocquard (1998). Paris : Martine Aboucaya et Yvon Lambert, 2013.

ROUBAUD, Jacques. Description du projet. Édité par Jean-Jacques Poucel, Caen : Nous, 2014.

ROUBAUD, Jacques. « Poésie et oralité ». Dire la poésie ?. Dir. Jean-François Puff. Paris : Cécile

Defaut, 2015. 307-318.

ROUBAUD, Jacques. Poétique. Remarques : poésie, mémoire, nombre, temps, rythme, contrainte, forme, etc. Paris : Seuil, 2016.

ROUBAUD, Jacques. Présentation générale / Quasi-Cristaux. URL : https://blogs.oulipo.net/qc/choixpremiere-partie/2-presentation-generale/ (page consultée le 13 mai 2021).

ROUBAUD, Jacques et Micaëla HENICH. Mille e tre, deux. 200 flèches. Courbevoie : Théâtre typographique, 1995.

ROUBAUD, Jacques et Jean-François PUFF. Roubaud : rencontre avec Jean-François Puff. Paris : Argol, 2008.

SAUZEDDE, Stéphane. « Avant-propos ». In octavo. Des formats de l'art. Dir. David Zerbib. Dijon :

ESAAA éditions ; Les Presses du Réel, 2015. 7-8.

ZERBIB, David. «Introduction : Voulez-vous enregistrer les modifications ». In octavo. Des formats de l'art. Dir. David Zerbib. Dijon : ESAAA éditions ; Les Presses du Réel, 2015. 13-19.

ZUMTHOR, Paul. Essai de poétique médiévale. Paris : Seuil, 1972.

\section{NOTES}

1. Le concept de «mouvance» a été défini par Paul Zumthor (Zumthor 507). Concernant la mouvance chez Jacques Roubaud, voir l'introduction de Jacques Roubaud médiéviste (Koble et Séguy 7-8).

2. Bernard Noël a souligné à sa manière dans L'Espace du poème cette continuité entre l'espace mental qui voit naître le poème et l'espace visuel: «J'ai fabriqué cette expression : 'l'espace du poème', parce que j'avais le sentiment que préexiste quelque chose. Avant d'écrire un poème, un livre, j'ai toujours essayé de faire exister une forme vide. Une forme qui n'a pas de forme, qui a des bords mais pas de limites [...] Voilà ce qui me préoccupe dans l'espace du poème». Plus loin, il souligne la nécessité de "préparer un lieu» qui fait advenir le poème. «Compter», «calculer» l'espace que 
doit occuper le poème (comme il a pu le faire pour le recueil La Chute des temps) et lui délimiter des «bords" est un des gestes évoqués pour «construire un espace qui donnera du sens à une précipitation verbale » (Noël 69-71).

3. Roubaud tente de saisir l'identité de la forme-sonnet au sein des multiples variations formelles à travers deux critères : l'exploration du champ des rimes et le jeu avec les proportions de la forme (Puff 2006).

4. Cette conception géométrique de la forme invite à s'interroger sur le rôle des blancs interstrophiques. Les dénombrer reviendrait à faire du sonnet une forme de 17 lignes agencées dans l'espace de la page, soit cette "installation de noir et de blanc» qu'évoque Roubaud dans le même poème. Emmanuel Hocquard affirmait quant à lui écrire des sonnets de "quatorze lignes », interrogeant le rôle des lignes blanches dans la définition de la forme : «Par exemple dans un sonnet découpé en quatre strophes, que faire des trois lignes blanches ? Faut-il les compter comme lignes ou les compter comme non-lignes? Je poserai un jour la question à Jacques Roubaud. En attendant ce jour, j'écris des sonnets de quatorze lignes qui se suivent. J'évite ainsi le problème posé par les strophes et les lignes blanches qui les séparent. Notez bien que les séparent soulève un autre débat. Peut-on dire que les strophes sont séparées les unes des autres par une ligne blanche? Non, bien sûr, on ne peut logiquement pas soutenir une chose pareille. Voilà une raison supplémentaire qui m'a fait adopter le sonnet de quatorze lignes qui se succèdent sans interruption. » (Hocquard, cité par Moncond'huy 2008, 19).

5. La citation de Hopkins est tirée de la Préface des poèmes publiés entre 1876 et 1889 (Hopkins 10) pour décrire une forme expérimentée dans trois poèmes intitulés : "Pied Beauty", "Peace", et "Ash Boughs". Les curtal sonnets sont ainsi composés de $10^{1 / 2}$ vers ( $6+4$ $1 / 2)$. Le dernier demi-vers constitue la «moitié » d'un pentamètre iambique. L'équation proposée montre que le sonnet court constitue une réduction de $3 / 4$ des proportions du sonnet pétrarquiste (Pitchford).

6. Nous remercions Jacques Roubaud et Marie-Louise Chapelle de nous avoir permis de consulter un manuscrit daté du 14/12/1965 offert à Alain Guérin, antérieur à celui qui avait été proposé à Raymond Queneau en vue de la publication chez Gallimard (fonds personnel de l'auteur). Un carnet des "sonnets préparatoires à Signe d'appartenance (1963-1966)» est déposé au Fonds Oulipo de la Bibliothèque de l'Arsenal. À notre connaissance, les sonnets manuscrits sur des quarts de feuilles, rangés autrefois dans une boîte à cigares, n'existent plus à ce jour.

7. «J'ai mis du lierre, des blancs ou des silences... », commente le poète après avoir évoqué les différentes variantes du sonnet et en particulier le sonnet court (Roubaud, Description du projet 34$)$.

8. « En donnant une représentation géométrique (en partie métaphorique) de la transformation qui les engendrait à partir de sonnets ordinaires, $\mathrm{j}$ 'ai voulu marquer que la distorsion qu'ils imposent n'est pas perceptible directement, ni rythmiquement, à l'oreille, ni visuellement, sur la page. [...] Elle est d'ordre conceptuel. Je ne cessai pourtant pas de voir ces poèmes comme des sonnets " ('le grand incendie de londres' 1678).

9. Le format $24 \times 32$ maintient ce rapport de proportion. Des extraits de ce manuscrit sont présentés dans le volume d'entretien précédemment cité (Roubaud et Puff 104 ; 106). 
10. Cette disposition est respectée pour la translittération en romaji des tankas empruntés aux grandes anthologies médiévales dans Mono no aware (Roubaud, Mono no aware).

11. Dans la suite de cet exposé, nous utilisons le terme de livre préféré par Roubaud à celui de « recueil » pour insister sur son organisation et son architecture internes. Il est pensé comme une forme abstraite à plusieurs dimensions.

12. Dans l'anthologie de sonnets Quasi-cristaux, récapitulant cinquante ans de recherches sur le sonnet, la métaphore du cristal - fréquemment employée de Sainte Beuve à Mallarmé - est employée pour désigner la forme (Roubaud, Quasi-cristaux). Pour marquer l'asymétrie et l'instabilité de nombre de ses propriétés formelles (Présentation générale / Quasi-Cristaux), Roubaud y ajoute le préfixe « quasi », convoquant ainsi un modèle mathématique - découvert par Daniel Shechtman, prix Nobel de chimie en 2011 - pouvant comporter jusqu'à six dimensions.

\section{RÉSUMÉS}

Le poète et mathématicien Jacques Roubaud s'est livré à une exploration de formes traditionnelles comme le sonnet et le tanka, pensées comme des formes en mouvement. Leur espace, géométrisé, est agencé selon un rapport de proportionnalité avec le format de la page. La potentialité de la forme agit ainsi sur le format. Toutefois, Roubaud conçoit le poème selon un "quatuor de formes»- deux externes (orale et écrite) et deux internes ("aurale », la voix intérieure et "éQrite», l'image mentale de la page) - au sein desquelles des écarts peuvent survenir. Enfin, le livre est pensé comme un hyperpoème en plusieurs dimensions, faisant intervenir la durée de déploiement dans la mémoire du lecteur. L'espace du poème excède donc la seule question du format pour tendre vers celle du volume, un espace méditatif dans lequel le poème est voué à résonner pour éviter son figement dans la seule forme écrite.

Poet and mathematician Jacques Roubaud has explored traditional forms such as the sonnet and the tanka, considered as forms in motion. Their geometrical space is arranged according to a relation of proportionality with the page. The potentiality of the form thus informs the format. Roubaud conceives the poem as a shifting "quartet of forms", of which two are external - spoken and written - and two are internal - "aural" (the inner voice) and "wRitten" (the mental picture of the page) -, and within which deviations can occur. The space of the book is thought of as a hyperpoem in several dimensions, involving the sense of duration in the reader's memory. The space of the poem therefore exceeds the sole question of format. It forms a volume, a meditative space in which the poem resonates, thus avoiding it being petrified in a purely written form.

\section{INDEX}

Mots-clés : Roubaud (Jacques), format, espace du poème, forme, sonnet, tanka, variation, mémoire, méditation

Keywords : Roubaud (Jacques), format, space, poetic form, sonnet, tanka, variation, memory, meditation 


\section{AUTEUR}

\section{MARGAUX COQUELLE-ROËHM}

Université de Poitiers - FoReLLIS

Ancienne étudiante de l'ENS Lyon, professeur agrégée de Lettres Modernes, Margaux CoquelleRoëhm est doctorante contractuelle à l'Université de Poitiers (laboratoire FoReLLIS) où elle enseigne la poésie contemporaine. Ses recherches portent sur les phénomènes de spatialité dans l'ensemble du corpus poétique de Jacques Roubaud, sous la direction de D. Moncond'huy. Elle s'intéresse également aux rapports entre littérature et photographie et à l'histoire des formes poétiques. Elle a publié plusieurs articles sur Roubaud et l'Oulipo et co-organisé la journée d'étude « Roubaud et l'anthologie » (Université de Poitiers, 22 novembre 2019) en présence de l'auteur. 